\title{
Archipel
}

ARCHIPEL Études interdisciplinaires sur le monde insulindien

$88 \mid 2014$

Varia

\section{Pilipinas Muna ! Filippiny prejdie sjevo ! K 80- ljetiou G.Y. Ratchkova (Pilipinas Muna! Les Philippines d'abord! En honneur du 80 anniversaire de Gennadiy Yevgenyevich Rachkov)}

Marlies Salazar

\section{OpenEdition}

Journals

Édition électronique

URL : http://journals.openedition.org/archipel/540

DOI : $10.4000 /$ archipel. 540

ISSN : 2104-3655

Éditeur

Association Archipel

Édition imprimée

Date de publication : 10 octobre 2014

Pagination : 221-224

ISBN : 978-2-910513-71-9

ISSN : 0044-8613

Référence électronique

Marlies Salazar, « Pilipinas Muna! Filippiny prejdie sjevo ! K 80-ljetiou G.Y. Ratchkova (Pilipinas Muna! Les Philippines d'abord! En honneur du 80 anniversaire de Gennadiy Yevgenyevich Rachkov) », Archipel [En ligne], 88 | 2014, mis en ligne le 10 septembre 2017, consulté le 25 septembre 2020. URL : http://journals.openedition.org/archipel/540; DOI : https://doi.org/10.4000/archipel.540 
Un mot sur l'édition du volume qui mérite d'être mentionnée : le beau papier, la mise en page aérée et le large format en rendent la lecture agréable. Une carte schématique (p. 12) situe les principaux groupes ethniques de la région du Mahakam. La bibliographie exhaustive sera particulièrement utile au chercheur comme au muséographe travaillant sur l'art et la culture matérielle de Bornéo.

\section{Antonio Guerreiro}

Maria Stanyukovich (ed.), Pilipinas Muna! Filippiny prejdie sjevo! K 80-ljetiou G.Y. Ratchkova (Pilipinas Muna! Les Philippines d'abord! En honneur du 80e anniversaire de Gennadiy Yevgenyevich Rachkov), série Maclay, vol. 4, Musée d'Anthropologie et d'Ethnologie, Académie Russe des Sciences, Saint Petersburg, 2011, 648 p., Ill., ISBN 978 5-88431-174-9

Ce volume est le fruit d'une conférence internationale pour célébrer le $80^{\mathrm{e}}$ anniversaire du linguiste Gennadiy Y. Rachkov. D'abord spécialiste de coréen, Rachkov s'est par la suite lancé dans l'étude du tagalog, puis fonda le Département de tagalog de l'École des Études Asiatiques et Africaines de l'Université de Saint Petersburg et le dirigea de 1967 à 2011. L'ouvrage rassemble des articles émanant d'experts en folklore, littérature, histoire, linguistique, anthropologie venus tant de Russie, que des Philippines et des États-Unis, ainsi que diverses bibliographies et catalogues de collections ethnographiques de Saint Petersbourg ayant rapport aux Philippines. Ce recueil, qui nous semble très intéressant, ne contient que quatre articles en anglais écrits respectivement par des Philippins et une Anglaise, aussi vaisje essayer de donner ici une idée du contenu en suivant le plan en quatre parties du recueil, afin de montrer la diversité et la richesse des études russes sur les Philippines.

\section{Les Philippines et la Russie}

Maria V. Stanyukovich dans son article «Les recherches sur les Philippines en Russie vues de Saint Petersburg » souligne l'importance de cette ville pour les études philippines. C'est de là que, à partir $\mathrm{du} \mathrm{XVIII}^{\mathrm{e}}$ siècle, sont partis tous les explorateurs russes, et les manuscrits et objets ethnographiques qu'ils ont rapportés s'y trouvent conservés, tant dans les archives qu'au Musée Ethnologique Pierre le Grand (Kunstkamera), le premier musée de Russie. Quant à la série de publications Maclay, elle tire son nom de l'anthropologue russe Nikolay N. Miklouho-Maclay qui, au XIX ${ }^{\mathrm{e}}$ siècle, voyagea en Nouvelle-Guinée et dans le Pacifique et se rendit trois fois aux Philippines, d'où il rapporta une riche collection d'objets toujours conservés dans ledit musée.

Ricardo Jose traite dans son article « La guerre russo-japonaise et les Philippines » de l'inspiration que la victoire nippone donna aux jeunes nationalistes philippins, dont le pays à peine débarrassé des Espagnols, venait d'être colonisé par les Américains.

Vladimir V. Noskov passe en revue les visites de la flotte russe à Manille du temps où l'empire russe essayait de s'ériger en force navale du Pacifique.

Mikhail Yu. Medvedev analyse les réformes récentes du Raja Muda Muedzul I Lail Tan Kiram du sultanat de Sulu, sud des Philippines, dans « Les symboles du sultanat royal de Sulu : héritage, contexte et réformes ». 


\section{Folklore et littérature}

Wystan De La Peña rend hommage à l'âge d'or de la littérature philippine en espagnol qui est à situer dans les années 1898-1941 de la période américaine.

Amparo Adelina C. Umali III décrit comment elle enseigne les chants Hudhud des Ifugao, la San Dionisio Komedya et la danse de l'aigle des Bontoc aux étudiants de l'Université des Philippines à Diliman, afin de sauver ces traditions.

Yuri E. Berezkin dans « Les Philippines et l'Indonésie » étudie quatre motifs typiques de la mythologie et du folklore et les met en rapport avec des données de la préhistoire sud-est-asiatique, ce afin de mieux les situer dans le temps et dans l'espace.

Sergey I. Yatsenko analyse l'épopée dite Biag ni Lam-ang « La vie de Lam-ang 》 des Ilocans et compare les traductions qui en ont été faites. Si on se réfère à la tradition russe des études folkloriques qui distingue deux genres d'épopées : geroicheskiy epos et bogatyrskaya skazka, le premier étant l'épopée d'un peuple et le deuxième l'histoire d'un héros individuel, on peut considérer « La vie de Lam-ang » comme appartenant plutôt au deuxième genre.

Artyom V. Kozmin compare la métrique des épopées ilokan et mongoles. Il arrive à la conclusion que les premières ont des contraintes formelles plus strictes concernant le nombre de syllables que peut contenir chaque ligne.

Sergey I. Yatsenko analyse la structure syllabique des vers et des rimes dans l'épopée ilokan Biag ni Lam-Ang ainsi que dans quarante chansons populaires et dix poèmes ilokan. Il est frappé par la structure mono-rythmique de la poésie ilokan.

Olga V. Koltyga présente l'ensemble des recherches philippines et russes sur le fameux poète philippin Francisco Balagtas et recommande de se concentrer, à l'avenir, davantage sur les analyses littéraires que sur les études socio-historiques.

\section{Linguistique}

Alexander K. Ogloblin observe le développement des formes du passif en tagalog, javanais, vieux malais et autres langues indonésiennes et conclut que le système du passif en tagalog est le plus archaïque et a conservé le plus grand nombre de formes, tandis que les autres langues ont réduit peu à peu les formes du passif ou même les ont complètement abandonnées.

Natalya F. Alieva compare la construction des voix active et passive dans les langues indonésiennes et le tagalog. Elle opte pour la thèse selon laquelle les relations entre actif et passif sont symétriques dans les langues indonésiennes, mais souligne qu'en tagalog les fomes passives sont mieux développées.

Yury I. Studenichnik examine la construction des verbes avec le préfixe mag- et l'infixe -um- en taglish, mélange de tagalog et d'anglais utilisé aux Philippines et surtout à Manille. En tagalog, il y a une différence sémantique entre les verbes construits avec mag- ou -um- qui a disparu en taglish. L'auteur essaie d'expliquer ce changement.

Sergey B. Klimenko traite du problème de savoir si le tagalog est une langue du type accusatif ou du type ergatif pour arriver à la conclusion préliminaire que le tagalog est un idiome ayant un système de voix symétriques.

\section{Anthropologie, Ethnologie, Ethnolinguistique}

Lawrence A. Reid aborde trois problèmes : d'abord, il essaie de définir les différentes significations du terme « indigène », surtout celle entre « indigène » et « minorité culturelle »; 
ensuite, il présente l'origine des langues du nord de Luzon sous la forme d'un arbre généalogique ; pour finir, il jette un coup d'œil sur la transformation des Bontok en ce qui concerne leur langue et leurs perspectives d'avenir. Il étudie ce groupe depuis cinquante ans et se lamente sur le fait que les jeunes, voulant aller à l'université et rêvant d'une vie en ville, ne s'intéressent plus à la langue et aux coutumes de leurs ancêtres. L'article de Lawrence Reid est le seul émanant d'un étranger à avoir été traduit en russe.

Ma. Crisanta N. Flores évoque une ancienne pratique encore vivante : le transport de produits faits en bambou et rotin sur des charettes tirées par des buffles depuis l'intérieur de la province de Pangasinan jusqu'à Manille. Cet archaïsme nous rappelle l'existence à l'époque préhistorique de l'État de Caboloan, à l'intérieur de ce qui est maintenant la province Pangasinan.

Elena G. Frolova décrit la situation linguistique contemporaine à Manille. Elle pense que le Filipino est la langue nationale des Philippines comme il est prescrit par les constitutions de 1897, 1935, 1946, 1974, 1987, et déplore que l'anglais soit toujours utilisé dans l'administration et dans la vie publique. Malheureusement Frolova se trompe parce que l'anglais est toujours une langue officielle des Philippines et, jusqu'en 1974, l'espagnol l'était aussi !

Yuri Yu. Krylov compare les vocabulaires austroasiatique et austronésien pour les métaux et conclut qu'il y a eu probablement des contacts linguistiques au cours de l'époque préhistorique.

Arina A. Lebedeva décrit les bateaux traditionnels des Philippines, de la pirogue jusqu'à l'embarcation à balancier, lesquels sont représentés par les dessins faits par le capitaine de corvette François Edmond Paris pendant le voyage autour du monde de l'Astrolabe, et publiés à Paris en 1841 .

Vadim R. Atnashev examine les droits constitutionnels aux Philippines et déplore les lacunes dans l'application des droits de l'homme, surtout ceux des groupes indigènes.

Igor Yu. Kotin retrace l'histoire des Indiens aux Philippines, traditionnellement appelés « Bumbai », du fait qu'ils partaient du port de Bombay. Leur arrivée est ancienne, mais leur présence n'est attestée dans les sources écrites qu'en 1762. Ils sont à présent quelque trente mille et la plupart sont d'origine sikh ou sindh.

Caroline Stone essaie de suivre les échanges culturels entre l'Amérique Latine et les Philippines dans le domaine des textiles. Elle cite trois exemples : l'ananas originaire du Brésil et du Paraguay qui a été transporté aux Philippines au $\mathrm{XVI}^{\mathrm{e}}$ siècle où l'on a développé une technique de tissage de la fibre et produit l'étoffe dite " piña », tandis que la technique de l'ikat, venant de l'Asie, se retrouve au Guatemala, et que les châles couverts de broderies dits « mantones de Manila » sont encore utilisés en Andalousie.

\section{Les Philippines dans l'art russe, dans les musées et les bibliothèques}

Cette dernière partie offre un inventaire des diverses collections conservées à St. Petersbourg:

- Publications sur les Philippines dans la bibliothèque de l'Institut des Manuscrits Orientaux. (Igor N. Wojewódzki),

- Collection de photographies de R.F. Barton conservée au Musée Ethnologique. Barton était un ethnographe américain qui a vécu à Saint Petersbourg de 1930 à 1940. Une partie de ses photographies se trouvent au musée. (Alexandra K. Kasatkina),

- Collections anciennes au Musée d'Ethnologie Pierre le Grand (Kunstkamera) de 
l'Académie des Sciences. (Vladimir N. Kislyako),

-Peintures faites par Mikhail Tikhanov, le premier peintre russe à avoir visité les Philippines, durant un voyage sur le bateau Kamchatka de 1817 à 1819. (Ekaterina V. Shilova)

- La série philippine des peintures de Vassiliy V. Vereschagin qui, en 1901, a représenté des soldats américains tués ou blessés pendant la guerre américano-philippine. (Julia S. Rutenko),

- Bibliographie de publications sur les Philippines au département d'Australie, d'Océanie et d'Indonésie du Musée Ethnologique. (Alexandra K. Kasatkina, ed.),

- Bibliographie des études philippines à St. Petersbourg ( $\mathrm{Xx}^{\mathrm{e}}$ et début $\mathrm{XxI}^{\mathrm{e}}$ siècle). (Maria V. Stanyukovich et Tatyana I. Shaskolskaya, ed.).

En bref, il semble qu'il y ait encore bien des trésors à découvrir à Saint Petersbourg !

Marlies Salazar

Hélène Bouvier (ed.), [avec les contributions de] Véronique Arnaud, Josiane Cauquelin, Dana Rappoport, L'art du Pathétique en Asie du Sud-Est Insulaire : Le choix des larmes, Paris, l'Harmattan, 2013, 248 p. ISBN : 978-2-343-01400-5.

L'ouvrage collectif coordonné et édité par Hélène Bouvier prolonge par une publication — et c'est là un grand mérite - une opération de recherche entreprise en 2007 intitulée « Pratiques littéraires et artistiques », qui a réuni sept chercheurs, membres du Centre Asie du Sud-Est (CASE, UMR8170, CNRS/EHESS).

Quatre brins pour tresser nos paroles symbolisent avec bonheur quatre cultures insulaires de 1'Asie du Sud-Est (deux à Taiwan et deux en Indonésie) sur lesquelles portent une interrogation commune et une réflexion particulière sur la notion privilégiée de « Pathétique ». L'ensemble amplifie nos connaissances et contribue à une anthropologie des émotions autour de la souffrance à partir de travaux monographiques menés sur un temps long par quatre femmes (trois ethnologues et une ethnomusicologue). La description ethnographique, associée à la pratique de la langue du groupe étudié, a commencé pour trois d'entre elles (Véronique Arnaud, Josiane Cauquelin, Hélène Bouvier) dans les années 1980, alors que nous étions les élèves de Georges Condominas au Laboratoire mixte du CéDRASEMI. Aborder une culture par la description rigoureuse des faits et par les paroles des hommes, était et reste la marque de l'exigeante méthode de celui qui fut notre Maître et que nous appelions avec affection « Condo $»^{5}$.

Partant de Taiwan au Nord et descendant vers les Petites Îles de la Sonde, les cultures exemplifiées par ces travaux nous offrent un nuancier d'émotions liées à la violence et les souffrances qu'elle implique sont vécues et perçues par les actants/acteurs, ainsi que le public ou les témoins présents dans le corps social.

5. Jusque dans les années 1990, nous avons eu l'occasion de travailler ensemble lors des séminaires de notre Centre et, après son démantèlement, lors d'un séminaire en ethno-poétique que j'ai animé dans un premier temps avec Tran Van Khê et qui a rassemblé des chants alternés en 13 langues et cultures de l'Asie du Sud-Est (continentale et insulaire). Il en émana une anthologie bilingue qui fut offerte en hommage à Jacques Dournes, Dam Bo, Chants alternés : Asie du Sud-Est (textes réunis par N. Revel, Paris : Sudestasie, 1992). Alors que j'avais entrepris en 2007-2008 un séminaire au Musée du quai Branly, Dana Rappoport s'est jointe à moi et nous avons pu mener une réflexion pluridisciplinaire et comparative sur les Littératures de la voix et les Arts de la Performance en Asie (2009-2014). 